\title{
Subtraction CTA: An Alternative Imaging Option for the Follow-Up of Flow-Diverter-Treated Aneurysms?
}

(D) M.P. Duarte Conde, (D)A.M. de Korte, (D).J.A. Meijer, (DR. Aquarius, (DH.D. Boogaarts, (D) R.H.M.A. Bartels, and (D). de Vries

\begin{abstract}
BACKGROUND AND PURPOSE: This was a pilot study to explore the diagnostic accuracy and safety of subtraction CTA combined with a single-energy metal artifact reduction algorithm (SEMAR) compared to DSA for the evaluation of intracranial aneurysm occlusion after flow diverter treatment.

MATERIALS AND METHODS: We included patients treated with a flow diverter for an unruptured intracranial aneurysm between November 2015 and November 2016. The patient cohort comprised 2 groups: those who underwent follow-up imaging 1 month after flow-diverter treatment and those with a known residual intracranial aneurysm after flow diverter treatment who underwent imaging at regular follow-ups. Full-brain subtraction CTA was performed on a 320-detector row CT system. A low-dose non-enhanced volume acquisition was followed by a contrast-enhanced volume CTA. Iterative and noise-reduction filters, SEMAR, and ${ }^{\text {SURE }}$ Subtraction algorithms were applied. DSA was performed on a flat panel C-arm angiography system. Standard posteroanterior, lateral, 3D, and detailed 2D acquisitions were performed. Imaging was independently scored by 2 clinicians. Aneurysm occlusion (Raymond scale) was our primary outcome parameter.
\end{abstract}

RESULTS: Thirteen intracranial aneurysms were evaluated with subtraction CTA and DSA. Nine aneurysm remnants were demonstrated by both subtraction CTA and DSA. The sensitivity and specificity of subtraction CTA for the detection of aneurysm occlusion were $100 \%$ (95\% Cl, 82.41\%-100\%) and 100\% (95\% Cl, 67.55\%-100\%), respectively. Agreement between readers was perfect $(\kappa=1.0)$. The smallest neck remnant detected on subtraction CTA was $1.2 \mathrm{~mm}$. No complications occurred.

CONCLUSIONS: Subtraction CTA with single-electron metal artifact reduction is effective in the reduction of metal artifacts of flow diverters and might therefore be a viable alternative in the assessment of intracranial aneurysm occlusion after flow diverter treatment.

ABBREVIATIONS: IA = intracranial aneurysm; FD = flow diverter; sCTA = subtraction CTA; SEMAR = single-electron metal artifact reduction

$\mathbf{F}$

ow diverters (FDs) are being increasingly used to treat complex intracranial aneurysms (IAs). ${ }^{1-3}$ These stentlike implants initially disrupt the blood flow into the IA and eventually facilitate parent artery reconstruction through endothelialization of the FD. Ideally this will lead to IA occlusion and rupture prevention., ${ }^{2,4}$

Although complete IA occlusion is the goal of the FD treatment, it is only achieved in around $75 \%-80 \%$ of cases. ${ }^{5}$ Identification of aneurysm remnants after FD treatment is therefore

Received May 13, 2018; accepted after revision August 9.

From the Departments of Neurosurgery (M.P.D.C., A.M.d.K., R.A., H.D.B., R.H.M.A.B., J.d.V.), Radiology and Nuclear Medicine (F.J.A.M.), and Pathology (R.A.), Radboud University Medical Center, Nijmegen, the Netherlands.

Mariana P. Duarte Conde and Antonius M. de Korte contributed equally to this article. This work was supported by Stryker Neurovascular.

Please address correspondence to A.M. de Korte, MD, Radboud University Medical Center, Department of Neurosurgery, PO Box 9101, 6500 HB Nijmegen, the Netherlands; e-mail: Thomas.dekorte@radboudumc.nl

http://dx.doi.org/10.3174/ajnr.A5817 important because remnants can still rupture and cause a subarachnoid hemorrhage. ${ }^{6}$ Digital subtraction angiography is considered the criterion standard to assess IA occlusion and IA remnants after FD treatment. However, this technique is invasive and has a low but not negligible complication rate..$^{7-9}$

Subtraction CT angiography (sCTA), in which a noncontrast acquisition is subtracted from a contrast-enhanced acquisition, might be a good alternative to DSA in the follow-up of patients treated with FDs. This technique has already been shown to produce results comparable with those of DSA in the detection and classification of IAs in the preoperative setting. ${ }^{10,11}$ Detection of small IAs and IAs adjacent to bony structures is even more accurate in sCTA. ${ }^{11}$ Additional advantages of sCTA compared with DSA are lower costs, ${ }^{12,13}$ a less invasive procedure, ${ }^{7-9}$ and a lower radiation dose for the patient. ${ }^{14}$ One major disadvantage of using SCTA in the follow-up of patients treated with FDs is that the metal wires of the implant can produce artifacts, which can hamper IA assessment.

With the introductions of new metal artifact-reduction algo- 


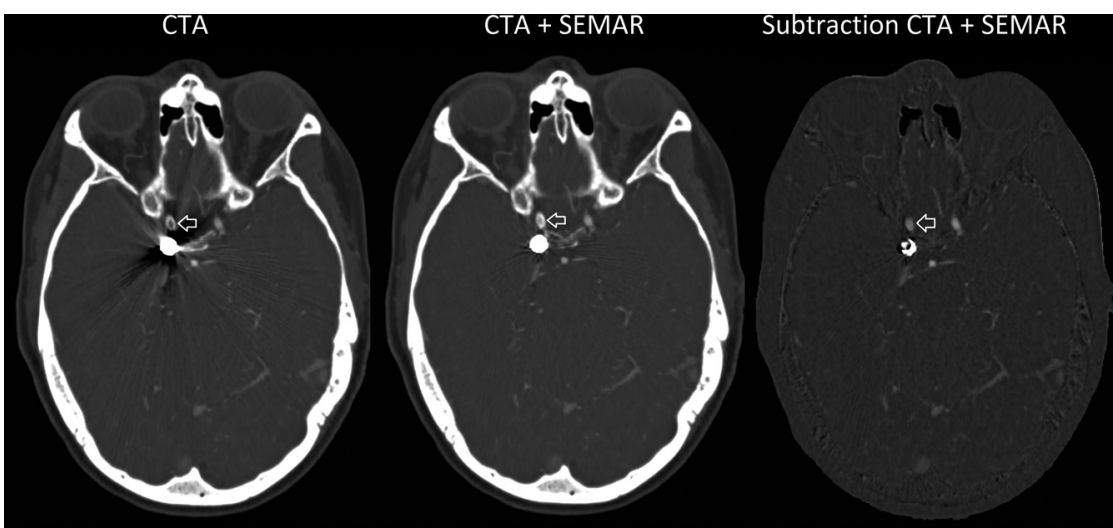

FIG 1. A patient with 3 aneurysms treated with either a flow diverter or coils. Axial view in which both a flow diverter (arrows) and coils are visible to illustrate the effect of single-energy metal artifact reduction. Non-SEMAR CTA (left), CTA with SEMAR (middle), and subtraction CTA with SEMAR (right) demonstrate the effects of SEMAR on metal artifacts.

rithms like single-energy metal artifact reduction (SEMAR) and subtraction algorithms ( ${ }^{\mathrm{SURE}}$ Subtraction; Toshiba Medical Systems, Tokyo, Japan) for second-generation 320-detector row CT scanners, ${ }^{15}$ it now seems that we might overcome this problem. The SEMAR algorithm uses several back-projection steps to extract metal objects from the raw data. Next, metal artifacts are filtered out by forwardprojection through ${ }^{\mathrm{SURE}}$ Subtraction algorithms. The corrected raw data are then reconstructed, and metal components without artifacts are placed back into the image. This algorithm, in combination with subtraction algorithms, has shown promising results regarding artifact reduction in several fields of medicine but has not been evaluated for assessing aneurysm occlusion after FD implantation. ${ }^{16-19}$

The goal of our study was to answer the following question: Can sCTA combined with a SEMAR algorithm effectively reduce metal artifacts of FDs, making it a viable alternative to DSA in the postoperative follow-up of patients treated with FDs? To answer our research question, we performed a pilot study to evaluate the diagnostic accuracy of sCTA compared with DSA for the evaluation of IA occlusion after FD placement.

\section{MATERIALS AND METHODS Study Population}

A pilot study was performed at our Department of Neurosurgery. Ethics committee approval was obtained, and all patients gave written informed consent. Patients treated with a FD for an unruptured IA were included in our series. The patient cohort comprised 2 groups: those who were treated between November 2015 and November 2016 receiving follow-up imaging 1 month after FD treatment and those with a known residual IA after earlier FD treatment who received imaging at regular follow-ups (which was between November 2015 and November 2016).

Exclusion criteria were the following: younger than 18 years of age, retreatment for a previously coiled IA, the presence of an $\mathrm{SAH}$, a known allergy or contraindication to the use of contrast agents, renal insufficiency, pregnancy, or the current clinical condition not allowing inclusion. Renal function was checked before ( $<3$ months) and 3 days after the procedure. sCTA and DSA were performed within 24 hours of each other.
Digital Subtraction Angiography

DSA was performed on a flat panel C-arm angiography system (Allura Xper FD20; Philips Healthcare, Best, the Netherlands). Before all acquisitions, a system-specific air calibration of the C-arm system was performed. Standard posteroanterior and lateral acquisitions were obtained using a diagnostic catheter via the transfemoral route. After standard 2D acquisitions, a $3 \mathrm{D}$ rotational acquisition was performed using a cerebral soft-tissue protocol (XperCT; Philips Healthcare) with selective contrast bolus injection via the ICA using a power injector (Angiomat Illumena; Covidien/Mallinckrodt, Lake Forest, California).

$3 \mathrm{D}$ reconstructions were generated and analyzed on a workstation (XtraVision; Philips Healthcare). 3D reconstructions were used to assess the best working projections for following detailed 2D acquisitions. At the end of the procedure, the arterial puncture wound was closed with an Angio-Seal (St. Jude Medical, Minnetonka, Minnesota) or by manual compression. An average of $50 \mathrm{~mL}$ of contrast material $(300 \mathrm{mg}$ of iodine $/ \mathrm{mL}$, iomeprol, Iomeron; Bracco, Milan, Italy) was used per procedure. The estimated radiation dose for the entire DSA (all acquisitions) was $10 \mathrm{mSv}$.

\section{Subtraction CTA}

Full-brain sCTA was performed on a wide-detector CT system (320-detector row CT scanner, Aquilion ONE Vision Edition; Toshiba Medical Systems). The scanning protocol included a lowdose nonenhanced volume acquisition at $120 \mathrm{kV}$ and $100 \mathrm{mAs}$ followed by a contrast-enhanced volume CTA at $120 \mathrm{kV}$ and 22 mAs using a bolus-tracked 50-mL contrast agent bolus (300 mg of Iomeron). Iterative and noise-reduction filters, SEMAR, and ${ }^{\mathrm{SURE}}$ Subtraction algorithms were applied (Fig 1). The estimated radiation dose was $2.6 \mathrm{mSv}$ as reported by the scanner after each acquisition.

\section{Image Analysis}

Studies were independently scored by 1 neuroradiologist (F.J.A.M., reader 1 with 12 years experience) and 1 neurosurgeon (M.P.D.C., reader 2 with 3 years experience). Neither of these 2 readers were involved in the treatment of the patients. Both sCTA and DSA studies were reviewed on a dedicated clinical workstation (Impax, Version 6.6; Agfa HealthCare, Mortsel, Belgium).

All variables were dichotomized to assess the diagnostic accuracy of sCTA compared with DSA as the reference standard. Intracranial aneurysm occlusion rate, based on the Raymond scale, was used as the primary study parameter and was categorized as either completely occluded (Raymond grade 1) or incompletely occluded (Raymond grade $\geq 2$ ).

We assessed the following secondary outcome measures: Device deployment, wall apposition, and neck coverage were scored as "complete" or "incomplete." Proximal and distal vessel patency 


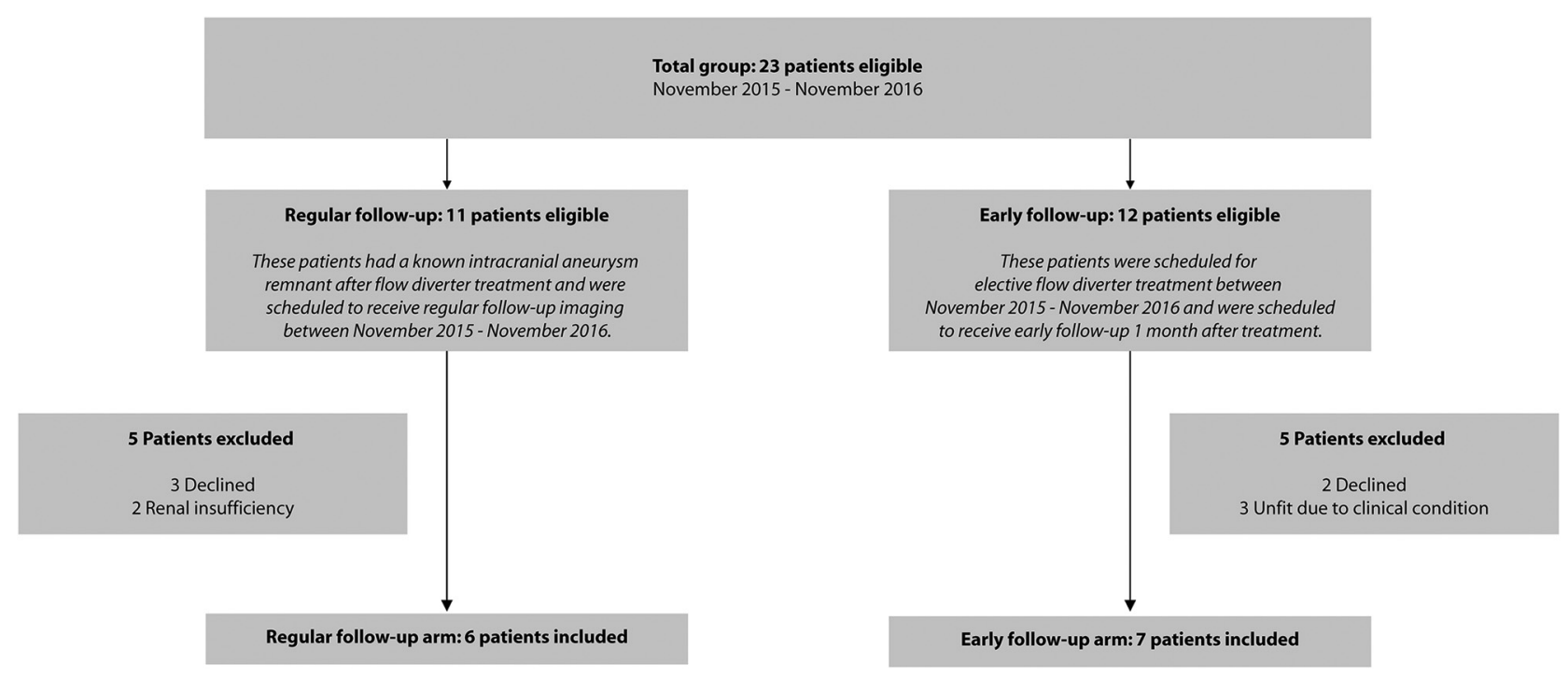

FIG 2. Description of study cohort.

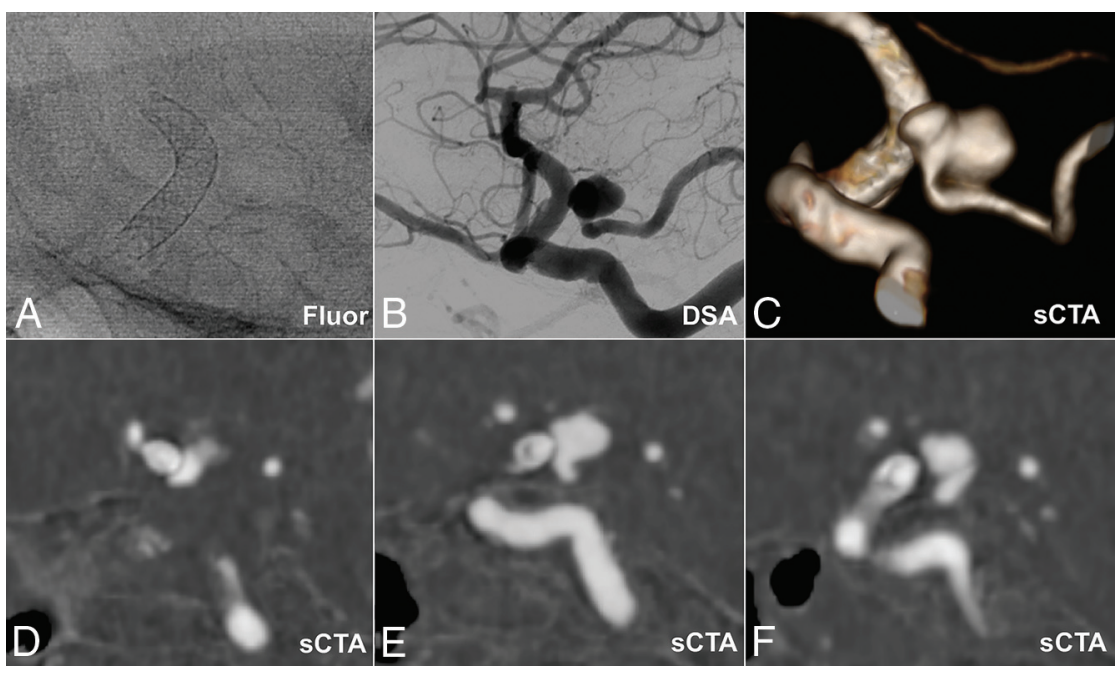

FIG 3. Residual aneurysm located at the origin of the posterior communicating artery after flow diverter placement in the internal carotid artery. Fluoroscopy $(A)$ with a flow diverter clearly visible, DSA $(B)$, subtraction CTA 3D view $(C)$, and subtraction CTA thin-slice 1-mm MIPs $(D-F)$.

was compared with pretreatment vessel diameter and scored as either "nonstenotic" ( $\leq 25 \%$ stenosis) or "stenotic" ( $>25 \%$ stenosis). Sidebranch patency was scored as "unchanged" or "changed" compared with pretreatment DSA.

Statistical analysis was performed on initial scoring. In case of discrepancies, readers were asked to re-evaluate these specific cases in a second reading to see whether consensus between sCTA and DSA scoring could be reached. These consensus readings were used solely for descriptive analysis and not for statistical analysis because of the great bias after the initial reading.

\section{Statistical Analysis}

Statistical analyses were performed using SPSS statistical software (Version 22.0; IBM, Armonk, New York). The sensitivity and specificity of sCTA compared with DSA regarding the primary outcome measure were calculated. $\kappa$ statistics were calculated for interrater reliability regarding the primary outcome measure. $\kappa$ values were scored according to the method of Fleiss et $\mathrm{al}^{20}:<0.4$, positive but poor agreement; 0.41-0.75, good agreement; and $>0.75$ excellent agreement. Descriptive statistics were used for patient and aneurysm characteristics and secondary parameters.

\section{RESULTS \\ Included Patients}

Between November 2015 and November 2016, twenty-three patients were treated with a flow diverter at our Department of Neurosurgery. Thirteen patients ( 8 women and 5 men) were enrolled in this study (Fig 2). Seven patients were newly treated and underwent early follow-up imaging 1 month after treatment to assess IA closure. The remaining 6 patients had a known IA remnant and were included at their regular follow-up moment. Five patients declined to participate in the study, and 2 patients were excluded due to their renal function. Three patients were excluded due to their clinical condition. These patients had neurologic deterioration after FD treatment either due to mass effect or ischemic events.

The average age of patients was $60 \pm 8$ years (range, 44-76 years). Treated IAs originated from the internal carotid artery $(54 \%, n=7)$, middle cerebral artery $(23 \%, n=3)$, posterior communicating artery $(8 \%, n=1)$, vertebral artery $(8 \%, n=1)$, and posterior inferior cerebellar artery $(8 \%, n=1)$.

All patients had been treated previously with a Surpass FD (Stryker Neurovascular, Kalamazoo, Michigan). Eleven patients were treated with $1 \mathrm{FD}$ and 2 patients were retreated with an additional FD due to persistent IA filling after the initial FD treatment. sCTA and DSA were performed within 24 hours of each other in every patient. No complications occurred.

AJNR Am J Neuroradiol 39:2051-56 Nov 2018 www.ajnr.org 


\section{Primary Outcome}

In all cases, sCTA and DSA provided adequate images for diagnostic evaluation. Nine incomplete IA occlusions were demonstrated by both sCTA and DSA (Figs 3-5).

The sensitivity and specificity of sCTA for the detection of IA occlusion were $100 \%$ (95\% CI, 82.41\%-100\%) and $100 \%$ (95\% CI, 67.55\%-100\%), respectively (Table). Agreement between readers was perfect $(\kappa=1.0)$. The smallest neck remnant detected on sCTA was $1.2 \mathrm{~mm}$ (Fig 6).

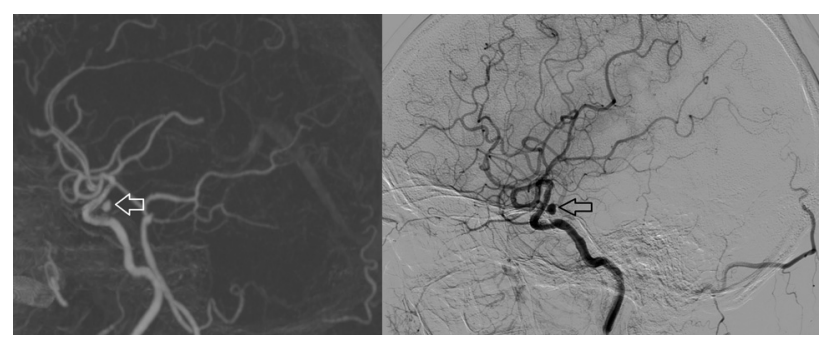

FIG 4. Residual aneurysm (arrows) located at the right carotid artery after flow-diverter placement in the internal carotid artery. Sagittal view of 45-mm-slice MIPs on subtraction CTA (left) and DSA (right).

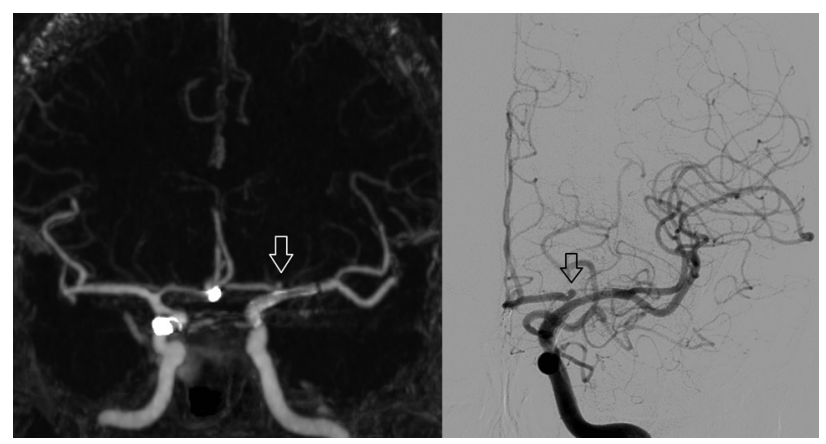

FIG 5. Residual aneurysm (arrows) located at the left anterior cerebral artery after flow-diverter placement in the internal carotid artery and middle cerebral artery. Coronal view of 20-mm-slice MIPs on subtraction CTA (left) and posteroanterior-acquisition DSA (right). Two previously coiled aneurysms on the contralateral carotid artery and anterior communicating artery can be seen on the subtraction CTA.

Number of discrepancies between SCTA and DSA for both readers

\begin{tabular}{|c|c|c|c|}
\hline \multirow[b]{2}{*}{ Intracranial Aneurysm Feature } & \multicolumn{2}{|c|}{$\begin{array}{l}\text { Discrepancies } \\
\text { between } \\
\text { sCTA and DSA }\end{array}$} & \multirow[b]{2}{*}{ Comments } \\
\hline & Reader 1 & Reader 2 & \\
\hline Aneurysm closure (Raymond 1) & 0 & 0 & NA \\
\hline Complete device deployment & 0 & 1 & $\begin{array}{l}\text { Device deployment was scored complete on sCTA but incomplete on } \\
\text { DSA; a second reading of this case confirmed complete device } \\
\text { deployment on DSA }\end{array}$ \\
\hline Complete wall apposition & 1 & 1 & $\begin{array}{l}\text { In } 1 \text { patient, both readers scored wall apposition incomplete on sCTA } \\
\text { but complete on DSA; a second reading of this case confirmed } \\
\text { incomplete wall apposition on DSA }\end{array}$ \\
\hline Complete neck coverage & 0 & 0 & NA \\
\hline Vessel patency distal (nonstenotic) & 1 & 0 & $\begin{array}{l}\text { Vessel patency was scored nonstenotic on sCTA but stenotic on DSA } \\
\text { in } 1 \text { case; a second reading confirmed nonstenosis on DSA }\end{array}$ \\
\hline Vessel patency proximal (nonstenotic) & 0 & 0 & NA \\
\hline Side branch patency (no change) & 0 & 1 & $\begin{array}{l}\text { In } 1 \text { case, a side branch was missed on follow-up sCTA; a second } \\
\text { reading of the case confirmed a patent side branch on SCTA }\end{array}$ \\
\hline
\end{tabular}

Note:-NA indicates not applicable 

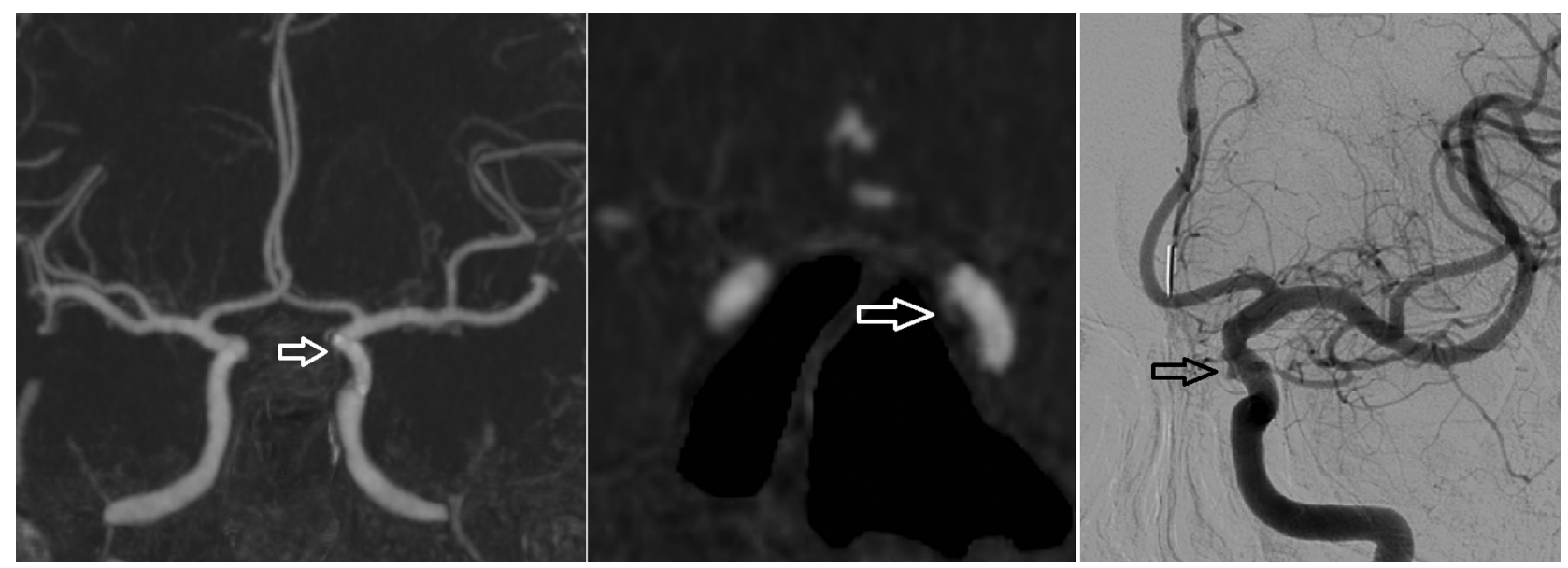

FIG 6. Small residual aneurysm (arrows) located at the left carotid artery after flow-diverter placement in the internal carotid artery. Coronal view of 20-mm-slice MIP on subtraction CTA (left), coronal subtraction CTA thin-slice 1-mm MIP (middle), and posteroanterior-acquisition DSA (right).

patients with dental implants ${ }^{26}$ and hip prostheses. ${ }^{17}$ Additionally, the SEMAR was also able to improve image quality in patients treated with coils for IA aneurysms and abdominal aneurysms (Fig 1). ${ }^{16,18}$, These results demonstrate the importance of SEMAR when using SCTA, and we think it might also be a useful tool for follow-up imaging when patients with IAs have been treated with surgical clips or detachable coils. This should be studied in future research.

This study has several limitations. First, because this was a pilot study, we used a small patient cohort and thus gathered limited data. However, we were able to collect a relatively high number of nonoccluded IAs by selecting patients with either a known aneurysm remnant as well as by performing a short follow-up after FD implantation. A large, prospective study is necessary to confirm the results of this pilot study.

Second, blinded scoring of sCTA and DSA studies would have improved the methodology of this study. However, with a limited study population, it will remain extremely difficult for readers to be completely blinded because they will likely recognize individual cases by the size, shape, and location of the IA.

Third, we only included patients treated with a Surpass FD device and results may not translate to other FDs because not all FDs are made from the same material. However, the cobalt chromium wires of the Surpass FD are expected to result in more pronounced metal artifacts compared with nitinol stents, which would make this a worst-case scenario.

\section{CONCLUSIONS}

Subtraction CTA with the SEMAR is effective in the reduction of metal artifacts of FDs and might, therefore, be a viable alternative in the assessment of IA occlusion after FD treatment.

Disclosures: J. de Vries - RELATED: Consultancy: Stryker Neurovascular.* H.D. Boogaarts - RELATED: Consultancy: Stryker Neurovascular.* *Money paid to the institution.

\section{REFERENCES}

1. Becske T, Kallmes DF, Saatci I, et al. Pipeline for uncoilable or failed aneurysms: results from a multicenter clinical trial. Radiology 2013; 267:858-68 CrossRef Medline

2. De Vries J, Boogaarts J, Van Norden A, et al. New generation of flow diverter (Surpass) for unruptured intracranial aneurysms: a prospective single-center study in 37 patients. Stroke 2013;44:1567-77 CrossRef Medline

3. Lylyk P, Miranda C, Ceratto R, et al. Curative endovascular reconstruction of cerebral aneurysms with the Pipeline embolization device: the Buenos Aires experience. Neurosurgery 2009;64:632-42; discussion 642-43; quiz N6 CrossRef Medline

4. Wakhloo AK, Gounis MJ. Revolution in aneurysm treatment: flow diversion to cure aneurysms: a paradigm shift. Neurosurgery 2014; 61(Suppl 1):111-20 CrossRef Medline

5. Brinjikji W, Murad MH, Lanzino G, et al. Endovascular treatment of intracranial aneurysms with flow diverters: a meta-analysis. Stroke 2013;44:442-47 CrossRef Medline

6. Feuerberg I, Lindquist C, Lindqvist M, et al. Natural history of postoperative aneurysm rests. J Neurosurg 1987;66:30-34 CrossRef Medline

7. Dawkins AA, Evans AL, Wattam J, et al. Complications of cerebral angiography: a prospective analysis of 2,924 consecutive procedures. Neuroradiology 2007;49:753-59 CrossRef Medline

8. Thiex R, Norbash AM, Frerichs KU. The safety of dedicated-team catheter-based diagnostic cerebral angiography in the era of advanced noninvasive imaging. AJNR Am J Neuroradiol 2010;31: 230-34 CrossRef Medline

9. Cloft HJ, Joseph GJ, Dion JE. Risk of cerebral angiography in patients with subarachnoid hemorrhage, cerebral aneurysm, and arteriovenous malformation: a meta-analysis. Stroke 1999;30:317-20 CrossRef Medline

10. Luo Z, Wang D, Sun X, et al. Comparison of the accuracy of subtraction CT angiography performed on 320-detector row volume CT with conventional $\mathrm{CT}$ angiography for diagnosis of intracranial aneurysms. Eur J Radiol;2012:81:118-22 CrossRef Medline

11. Ramasundara S, Mitchell PJ, Dowling RJ. Bone subtraction CT angiography for the detection of intracranial aneurysms. J Med Imaging Radiat Oncol 2010;54:526-33 CrossRef Medline

12. Harrison MJ, Johnson BA, Gardner GM, et al. Preliminary results on the management of unruptured intracranial aneurysms with magnetic resonance angiography and computed tomographic angiography. Neurosurgery 1997;40:947-55; discussion 955-57 CrossRef Medline

13. Dehdashti AR, Binaghi S, Uske A, et al. Comparison of multislice computerized tomography angiography and digital subtraction angiography in the postoperative evaluation of patients with clipped aneurysms. J Neurosurg 2006;104:395-403 CrossRef Medline

14. Manninen AL, Isokangas JM, Karttunen A, et al. A comparison of radiation exposure between diagnostic CTA and DSA examina-

AJNR Am J Neuroradiol 39:2051-56 Nov 2018 www.ajnr.org 
tions of cerebral and cervicocerebral vessels. AJNR Am J Neuroradiol 2012;33:2038-42 CrossRef Medline

15. Golshani B, Lazzaro MA, Raslau F, et al. Surveillance imaging after intracranial stent implantation: non-invasive imaging compared with digital subtraction angiography. Neurointerv Surg 2013;5: 361-65 CrossRef Medline

16. Kidoh M, Utsunomiya D, Ikeda O, et al. Reduction of metallic coil artefacts in computed tomography body imaging: effects of a new single-energy metal artefact reduction algorithm. Eur Radiol 2016; 26:1378-86 CrossRef Medline

17. Yasaka K, Maeda E, Hanaoka S, et al. Single-energy metal artifact reduction for helical computed tomography of the pelvis in patients with metal hip prostheses. Jpn J Radiol 2016;34:1-8 CrossRef Medline

18. Pan YN, Chen G, Li AJ, et al. Reduction of metallic artifacts of the post-treatment intracranial aneurysms: effects of single energy metal artifact reduction algorithm. Clin Neuroradiol 2017 Nov 16. [Epub ahead of print] CrossRef Medline

19. Asano $Y$, Tada A, Shinya T, et al. Utility of second - generation singleenergy metal artifact reduction in helical lung computed tomography for patients with pulmonary arteriovenous malformation after coil embolization. Jpn J Radiol 2018;36:285-294 CrossRef Medline

20. Fleiss J, Levin B, Cho Paik M. Statistical Methods for Rates and Proportions. Hoboken: John Wiley Sons; 2003:800

21. Attali J, Benaissa A, Soize S, et al. Follow-up of intracranial aneurysms treated by flow diverter: comparison of three-dimensional time-of-flight MR angiography (3D-TOF-MRA) and contrast-enhanced MR angiography (CE-MRA) sequences with digital subtraction angiography as the gold standard. J Neurointerv Surg 2016;8: 81-86 CrossRef Medline

22. Toni F, Marliani AF, Cirillo L, et al. 3T MRI in the evaluation of brain aneurysms treated with flow-diverting stents: preliminary experience. Neuroradiol J 2009;22:588-99 CrossRef Medline

23. Patzig M, Forbrig R, Ertl L, et al. Intracranial aneurysms treated by flow-diverting stents: long-term follow-up with contrast-enhanced magnetic resonance angiography. Cardiovasc Intervent Radiol 2017; 40:1713-22 CrossRef Medline

24. Yu SC, Lee KT, Lau TW, et al. Intravenous C-arm conebeam CT angiography following long-term flow-diverter implantation: technologic evaluation and preliminary results. AJNR Am J Neuroradiol 2016;37:481-86 CrossRef Medline

25. Yang P, Ahmed A, Schafer S, et al. Low-dose volume-of-interest Carm CT imaging of intracranial stents and flow diverters. AJNR Am J Neuroradiol 2016;37:648-54 CrossRef Medline

26. Yasaka K, Kamiya K, Irie R, et al. Metal artefact reduction for patients with metallic dental fillings in helical neck computed tomography: comparison of adaptive iterative dose reduction 3D (AIDR 3D), forward-projected model-based iterative reconstruction solution (FIRST) and AIDR 3D with single-energy metal artefact reduction (SEMAR). Dentomaxillofac Radiol 2016;45:20160114 CrossRef Medline 